\title{
Tetra(p-tolyl)borate-Functionalized Solvent Polymeric Membrane: A Facile and Sensitive Sensing Platform for Peroxidase and Peroxidase Mimetics
}

\author{
Xuewei Wang ${ }^{[a, b]}$ and Wei Qin*[a]
}

\begin{abstract}
The determination of peroxidase activities is the basis for enzymelabeled bioaffinity assays, peroxidasemimicking DNAzymes- and nanoparticles-based assays, and characterization of the catalytic functions of peroxidase mimetics. Here, a facile, sensitive, and cost-effective solvent polymeric membrane-based peroxidase detection platform is described that utilizes reaction intermediates with different $\mathrm{p} K_{\mathrm{a}}$ values from those of substrates and final products. Several key but long-debated intermediates in the peroxidative oxidation of $o$-phenylenediamine $(o$-PD)
\end{abstract}

have been identified and their charge states have been estimated. By using a solvent polymeric membrane functionalized by an appropriate substituted tetraphenylborate as a receptor, those cationic intermediates could be transferred into the membrane from the aqueous phase to induce a large cationic potential response. Thus, the poten-

Keywords: biosensors • intermediate-sensitive membranes - peroxidase $\cdot$ peroxidase mimetics potentiometry tiometric indication of the $o$-PD oxidation catalyzed by peroxidase or its mimetics can be fulfilled. Horseradish peroxidase has been detected with a detection limit at least two orders of magnitude lower than those obtained by spectrophotometric techniques and traditional membrane-based methods. As an example of peroxidase mimetics, G-quadruplex DNAzymes were probed by the intermediate-sensitive membrane and a label-free thrombin detection protocol was developed based on the catalytic activity of the thrombinbinding G-quadruplex aptamer.

\section{Introduction}

Peroxidase and its mimetics have a wide range of applications in the area of analytical science and environmental engineering. ${ }^{[1]}$ In immunoassays, nucleic acid hybridization assays, and molecular imprinting polymer-based sensing, horseradish peroxidase (HRP), peroxidase-mimicking DNAzymes and nanoparticles have been applied as highly efficient amplifying labels. ${ }^{[1,2]}$ By combining oxidases with peroxidase or its mimetics, a large number of analytes such as glucose ${ }^{[3]}$ melamine, ${ }^{[4]}$ and organophosphates ${ }^{[5]}$ could be detected. Also, the peroxidatic activities of magnetic nanoparticles and carbon materials have been utilized to detect disease-associated single nucleotide polymorphism and quantify cancer cells. ${ }^{[6]}$ In environmental applications, there is also

[a] X. Wang, Prof. W. Qin

Key Laboratory of Coastal Zone

Environmental Processes and Ecological Remediation

Yantai Institute of Coastal Zone Research (YIC)

Chinese Academy of Sciences (CAS)

Shandong Provincial Key Laboratory of

Coastal Zone Environmental Processes

YICCAS, Yantai Shandong 264003 (P.R. China)

Fax: $(+86) 5352109000$

E-mail:wqin@yic.ac.cn

[b] X. Wang

University of Chinese Academy of Sciences Beijing 100049 (P.R. China)

Supporting information for this article is available on the WWW under http://dx.doi.org/10.1002/chem.201300284. great interest in the use of peroxidase and its mimetics as green catalysts for the degradation of organic pollutants. ${ }^{[1]}$

The increasing investigations and applications of peroxidase and its mimetics leads to a great demand for efficient techniques measuring peroxidase activities, which are essential both to transduce the peroxidase activity-involved biorecognition events and to characterize the catalytic functions of peroxidase mimetics. To this end, optical methods based on UV/Vis absorbance, ${ }^{[6,7]}$ fluorescence, ${ }^{[8]}$ and chemiluminescence ${ }^{[9]}$ are commonly used. However, these techniques generally require complex instruments and software, which hinders their miniaturization and out-of-lab applications, and also increases the test cost. Moreover, the color and turbidity in biological fluids and nanoparticle solutions can interfere with the optical measurements and additional separation steps such as centrifugation may be required. ${ }^{[4,10]}$

To overcome these limitations, electrochemical approaches can be employed. The solvent polymeric membrane (SPM)-based ion-selective electrodes represent an important group of electrochemical sensors with attractive features. ${ }^{[11,12]}$ The preparation of solvent polymeric membrane electrodes (SPMEs) is easy and their costs are fairly low. They are easy to be miniaturized and integrated, and resistant to color and turbid interferences, which are important for biomedical analyses. The potentiometer is simpler than optical measurement instruments and other electrochemical instruments. With these advantages, ion-selective SPMEs have become the standard technique in blood electrolyte analysis $^{[11]}$ and a powerful tool for measurements of intracellular and extracellular ions. ${ }^{[13]}$ In addition, by using potential 
signals from the generation of ionic products or the consumption of ionic substrates in enzymatic reactions, various enzymes such as cholinesterase ${ }^{[14]}$ galactosidase, ${ }^{[15]}$ and protease $^{[16]}$ have been indirectly detected by ion-selective SPMEs. However, for peroxidase-catalyzed reactions, ion-selective SPMEs are rarely used primarily owing to the lack of ionic substrates and products detectable by SPMs. ${ }^{[15,17]}$

Traditionally, for a reaction without ionic reactants and products detectable by a SPM, the possibility of potentiometric indication by ion-selective SPMEs would be precluded. In a recent short communication, a biosensing system based on potential responses of reaction intermediates rather than chemically stable substrates or final products was demonstrated for the first time. ${ }^{[18]}$ The one-electron oxidations of tertiary amine structures in $N, N^{\prime}, N, N^{\prime}$-tetramethylbenzidine $\left(N_{4}\right.$-TMB) produce intermediates including a tertiary amine cation radical and an iminium ion, which are intrinsically positively charged independent of the $\mathrm{pH}$ and could be detected by the ion-selective SPME. Although intrinsically charged intermediates are not common for other enzymatic reactions, the intermediate-based design concept opens a new avenue for sensing of enzymatic reactions that seem undetectable by ion-selective SPMEs from a one-step "substrate to product" perspective.

In this paper, based on the peroxidase-catalyzed oxidation reaction of $o$-phenylenediamine ( $o$-PD), we demonstrate the utility of $\mathrm{p} K_{\mathrm{a}}$ differences between intermediates and chemically stable substrates and final products for potentiometric biosensing. Intermediates in this reaction have been found to show much larger $\mathrm{p} K_{\mathrm{a}}$ values than the substrate and final product, and are mainly cationic under near-neutral $\mathrm{pH}$ conditions. By using SPMEs doped with an appropriate substituted tetraphenylborate salt as the intermediate receptor, the potential responses of these intermediates can be obtained, which enables the sensitive detection of horseradish peroxidase (HRP) and peroxidase mimetics such as G-quadruplex/hemin DNAzymes.

\section{Results and Discussion}

The main product of the $\mathrm{H}_{2} \mathrm{O}_{2}$-mediated oxidation of $o$-PD catalyzed by HRP has been identified to be 2,3-diaminophenazine (DAP) ${ }^{[19]}$ By using the visible absorbance, ${ }^{[20]}$ fluorescence, ${ }^{[21]}$ chemiluminescence, ${ }^{[22]}$ and redox behavior ${ }^{[23]}$ of DAP, this reaction has been transduced by various optical and electrochemical techniques, and corresponding peroxidase detection protocols have been developed. According to the $\mathrm{p} K_{\mathrm{a}}$ of DAP (5.1), ${ }^{[24]}$ it exists mainly in the electrically neutral form at the optimal pH of HRP (6.0 to 6.5). ${ }^{[25]}$ Thus, DAP is unexpected to induce a large potential response on an ion-selective SPM. $o$-PD with a similar $\mathrm{p} K_{\mathrm{a}}$ of $4.6^{[26]}$ is also dominatingly nonionic and could not yield a large potential signal. Therefore, the HRP-catalyzed oxidation of $o$ $\mathrm{PD}$ is considered undetectable by the ion-selective SPME from the traditional sensing methodology based on the potential response of the substrate or the final product.
Indeed, the potential response of the HRP-catalyzed $o$-PD oxidation on SPMs has not been reported although the reaction has been well-known and widely used for long.

Identification of intermediates in the peroxidase-catalyzed $\boldsymbol{o}$-PD oxidation: With a view to exploring the possibility of monitoring the peroxidase-catalyzed $o$-PD oxidation by an ion-selective SPM, the intermediates generated in the $o$-PD peroxidative oxidation were examined. As a basic step for the HRP-catalyzed oxidations of arylamines, the aromatic substrates are first oxidized into their free radicals through single electron-transfer. ${ }^{[27]}$ However, for intermediates other than the $o$-PD radical, there are conflicting reports. Firstly, the existence of another monomer intermediate, $o$-benzoquinone diimine (BQD) is controversial. $o$-PD radicals were assumed to directly couple into oligomers in some studies, ${ }^{[19 a, 28]}$ whereas BQD was proposed to be generated from the $o$-PD radical and responsible for the subsequent condensation reactions in other reports. ${ }^{[19 b, 29]}$ Here, the involvement of BQD in the peroxidase-catalyzed $o$-PD oxidation was experimentally confirmed by using butylated hydroxyanisole (BHA), a specific trapping agent for diimine. ${ }^{[30]}$ Upon addition of BHA into the reaction mixture of $o$-PD oxidation, an adduct of BQD with BHA was clearly observed in the mass spectrum (Figure S1 and Scheme S1 in the Supporting Information). Since the arylamine radical cannot form an adduct with $\mathrm{BHA},{ }^{[30 \mathrm{~b}]}$ the existence of $\mathrm{BQD}$ can be confirmed in the HRP-catalyzed $o$-PD oxidation.

The first condensation intermediate in this reaction is a dimer with a pseudo-molecular peak at $\mathrm{m} / \mathrm{z} 213$ in the mass spectrum (Figure 1). Such a dimer has been widely accepted as the precursor of DAP. However, the structure of this dimer has not been experimentally determined, although it has been suggested to be 2-amino- $N$-(2-aminophenyl)- $p$ benzoquinone diimine $(\mathrm{AABI})^{[31]}$ or 5,10-dihydro-2,3-diaminophenazine (DDAP) ${ }^{[32]}$ Here, experimental evidence was found that supports the existence of AABI rather than DDAP. Firstly, in analogy to BQD, this dimer can also form an adduct with BHA (Figure S1 and Scheme S1 in the Supporting Information), which indicates the existence of a diimine structure. Secondly, consecutive losses of two $\mathrm{NH}_{3}$ were observed from the fragmentation pattern at $\mathrm{m} / \mathrm{z} 213$ $(213 \rightarrow 196 \rightarrow 179$; Figure S2 in the Supporting Information). Since two consecutive $\mathrm{NH}_{3}$ losses (loss of 17) cannot occur on the same benzene ring in the sequential mass spectrometry, ${ }^{[28]}$ only AABI could rationalize these fragmentations. This structure can be reasonably formed by the 1,4-addition of amino group of $o$-PD to the quinoid ring of BQD and converted into DAP by two-electron oxidation and intramolecular cyclization. ${ }^{[33]}$

In addition to the dimer intermediate, trimers and tetramers were also observed for the $o$-PD oxidation under various oxidation conditions. Figure $1 \mathrm{~A}$ and Figure $1 \mathrm{~B}$ show the mass spectra for the $o$-PD oxidations catalyzed by HRP $\left(0.1 \mathrm{UmL}^{-1}\right)$ in the presence of an excess of $\mathrm{H}_{2} \mathrm{O}_{2}$ and an equimolar concentration of $\mathrm{H}_{2} \mathrm{O}_{2}$, respectively. As can be seen, trimers at $\mathrm{m} / \mathrm{z}, 319$ and 317 , as well as tetramers at $\mathrm{m} / \mathrm{z}$ 


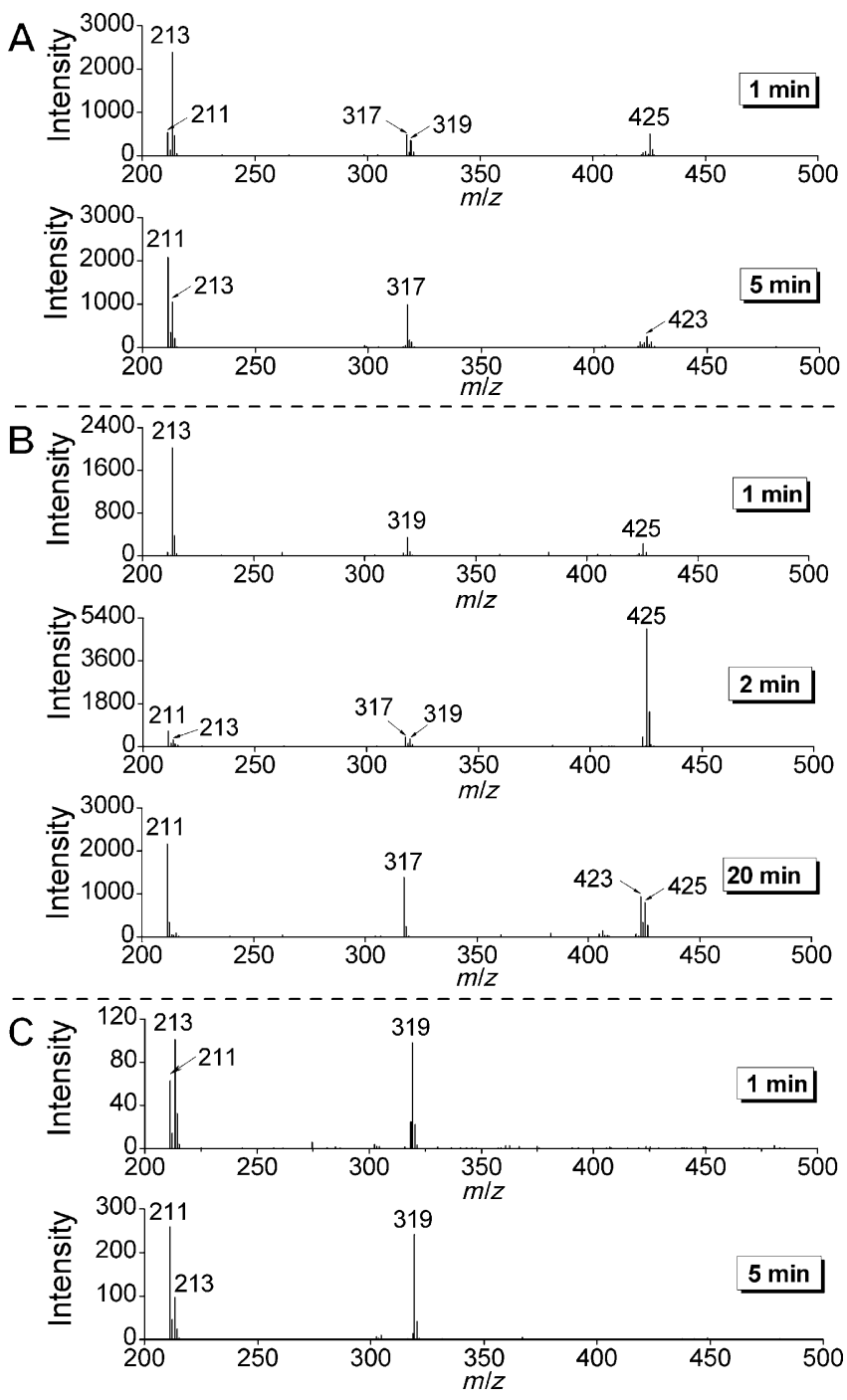

Figure 1. Mass spectra for the oxidations of $o-\mathrm{PD}(1 \mathrm{mM})$ at $\mathrm{pH} 6.0$ with A) $\mathrm{H}_{2} \mathrm{O}_{2}(5 \mathrm{~mm})$ and $\mathrm{HRP}\left(0.1 \mathrm{UmL}^{-1}\right)$, B) $\mathrm{H}_{2} \mathrm{O}_{2}(1 \mathrm{~mm})$ and HRP (0.1 $\left.\mathrm{UmL}^{-1}\right)$, and C) $\mathrm{H}_{2} \mathrm{O}_{2}(1 \mathrm{~mm})$ and $\mathrm{HRP}\left(10^{-3} \mathrm{U} \mathrm{mL}^{-1}\right)$.

425 and 423 were generated in both cases, although their generation and degradation rates differ. With a lower concentration of $\mathrm{H}_{2} \mathrm{O}_{2}$ (e.g., $0.2 \mathrm{~mm}$ ), the behaviors of intermediates (data not shown) are analogous to those with an equimolar concentration of $\mathrm{H}_{2} \mathrm{O}_{2}$ (Figure $1 \mathrm{~B}$ ). However, when the activity of HRP was decreased to $10^{-3} \mathrm{U} \mathrm{mL}^{-1}$ or lower, the trimer at $\mathrm{m} / z 319$ was observed, but no other trimer and tetramer intermediates were detected (Figure $1 \mathrm{C}$ ). Moreover, the variation of $\mathrm{H}_{2} \mathrm{O}_{2}$ concentration does not significantly change the intermediate behaviors, in contrast to the case with $0.1 \mathrm{U} \mathrm{mL}^{-1} \mathrm{HRP}$.

Based on these MS data in combination with sequential MS characteristics of $o$-PD oxidation intermediates, the structures and generation routes of these trimeric and tetrameric intermediates are proposed in Scheme 1. The trimer at $m / z 317$ can be formed by condensation of AABI and $\mathrm{BQD}$ in the same way as that of $o$-PD and BQD. For another trimer at $\mathrm{m} / \mathrm{z} 319$, it is likely to result from the addition

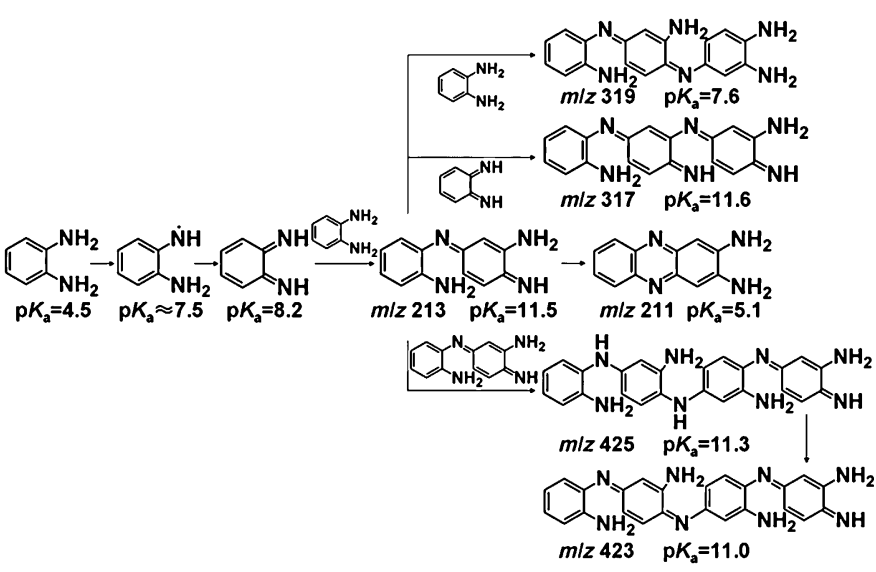

Scheme 1. Proposed generation pathways of intermediates in the HRPcatalyzed $o$-PD oxidation, and the pseudo-molecular ion peaks and $\mathrm{p} K_{\mathrm{a}}$ values $^{[34]}$ of these intermediates.

of the terminal imino group of AABI to the benzene ring of $o$-PD. The $o$-PD-involved generation route of the trimer at $\mathrm{m} / \mathrm{z} 319$ is corroborated by its enhanced formation in the presence of low concentrations of HRP (Figure 1C), because the low oxidation rate renders residual $o$-PD abundant. Also, the resulting structure could well account for three consecutive $\mathrm{NH}_{3}$ losses $(319 \rightarrow 302 \rightarrow 285 \rightarrow 268)$ in sequential MS, whereas other candidate structures bearing the dihydrophenazine moiety (like DDAP) also with a molecular weight of 318 could not rationalize these fragments. ${ }^{[28]}$

According to Figure $1 \mathrm{~B}$, the tetramer intermediate at $\mathrm{m} / \mathrm{z}$ 425 is generated concomitantly with a sudden drop of the peak at $m / z$ 213. This tetramer presumably results from the coupling of two AABI molecules, which could induced by the electrophilic attack of protonated imino group of one $\mathrm{AABI}$ on the benzene ring of the other AABI, and the subsequent intramolecular hydrogen migration. With further oxidation, it is converted into another tetramer at $\mathrm{m} / \mathrm{z} 423$. These consecutive processes including electrophilic attack, hydrogen migration, and oxidation have been observed in the reaction of $p$-benzoquinone diimine with aniline ${ }^{[35]}$ and $m$-phenylenediamine. ${ }^{[36]}$ In addition, the fragmentation patterns of two tetramers (four consecutive losses of $\mathrm{NH}_{3}$ for both tetramers) also confirm that these tetramers are indeed polyaniline-like linear structures without the moieties of phenazine or dihydrophenazine.

With a longer reaction period, various intermediates would transform into the final products. However, since these degradations occur primarily in a time scale beyond the potentiometric measurements in biosensing applications, efforts on characterizing and interpreting the degradation processes of these intermediates were not made in this work.

Potential responses of reaction intermediates on the SPM with an intermediate receptor: According to the $\mathrm{p} K_{\mathrm{a}}$ values of the seven intermediates shown in Scheme 1, they are generally positively charged under near-neutral $\mathrm{pH}$ conditions. 
By using a SPM functionalized with an appropriate receptor of aromatic cations, these cationic intermediates are expected to induce potential responses through an assisted phasetransfer process. To demonstrate the contribution of intermediates, the potential response of the HRP-catalyzed $o$-PD oxidation on a tetra( $p$-tolyl)borate $\left(\mathrm{TTB}^{-}\right)$-doped SPME in a phosphate buffer solution (PBS) at $\mathrm{pH} 7.5$ was measured. At this $\mathrm{pH}, o$-PD and DAP are both predominantly uncharged $(99.9 \%$ for $o-\mathrm{PD}$ and $99.6 \%$ for DAP) and, thus, do not contribute to potential responses. As shown in Figure 2, a large real-time potential response (-) was

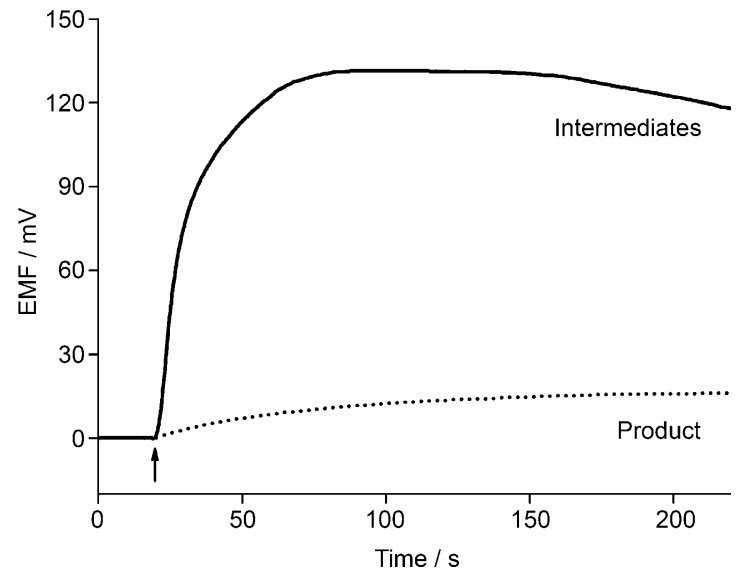

Figure 2. Potential responses to the final product $(\cdots .$.$) and to the inter-$ mediates (-) upon addition of $\operatorname{HRP}\left(0.1 \mathrm{U} \mathrm{mL}^{-1}\right)$ into PBS $(50 \mathrm{~mm})$ of $\mathrm{pH} 7.5$ containing $o$-PD $(0.05 \mathrm{~mm})$ and $\mathrm{H}_{2} \mathrm{O}_{2}(0.25 \mathrm{~mm})$. For the preparation of the final product, a PBS $(50 \mathrm{~mm}, \mathrm{pH} 7.5)$ containing $o$-PD $(0.05 \mathrm{~mm}), \mathrm{H}_{2} \mathrm{O}_{2}(0.25 \mathrm{~mm})$, and $\mathrm{HRP}\left(0.1 \mathrm{UmL}^{-1}\right)$ was allowed to react for $1 \mathrm{~h}$ before the measurement.

indeed obtained upon addition of HRP to the mixture of $o$ $\mathrm{PD}$ and $\mathrm{H}_{2} \mathrm{O}_{2}$, whereas a rather small potential response $(. . .$.$) was observed when o$-PD was dominatingly oxidized into DAP (the conversion of $o$-PD into DAP was confirmed by its pseudo-molecular ion peak at $\mathrm{m} / \mathrm{z} 211$ in the mass spectrum). Experiments also show that no significant potential responses can be obtained when any of $o$-PD, HRP, and $\mathrm{H}_{2} \mathrm{O}_{2}$ is absent. Therefore, the real-time potential response toward the HRP-catalyzed oxidation of $o$-PD should be attributed to the $o$-PD oxidation intermediates rather than the final product DAP or any reactant. Moreover, for the realtime potential response in Figure 2, an obvious potential drop was observed following the cationic potential response (the potential can further decrease over extended time), which also confirms that potential responses originate from the chemically unstable species.

Optimization of the intermediate-sensitive SPM and its application in HRP detection: Substituted tetraphenylborates were used as the intermediate receptors because they have been found to form complexes with aromatic nitrogen-centered cations through noncovalent interactions including hydrogen bonding, $\pi-\pi$, and cation $-\pi$ interactions. ${ }^{[37]}$ Figure 3

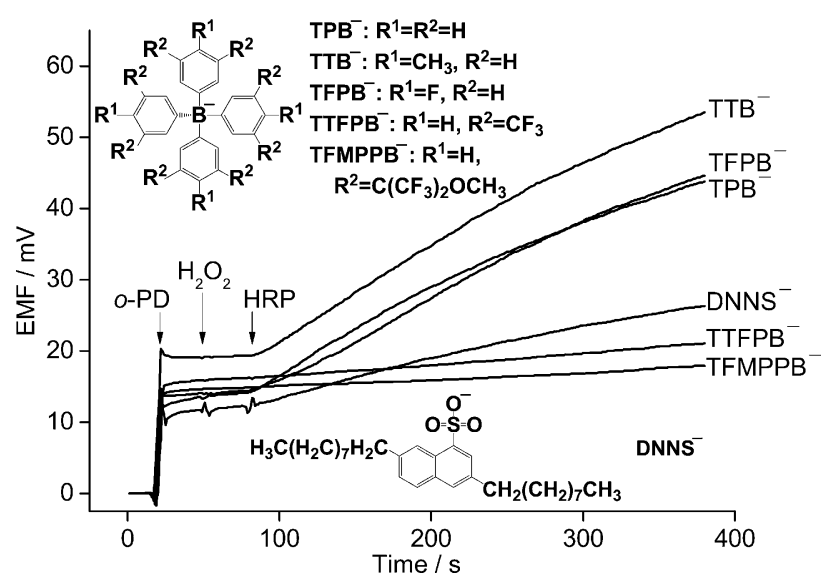

Figure 3. Potential responses of polymeric membranes doped with different receptors $\left(25 \mathrm{~mm} \mathrm{~kg}^{-1}\right)$ toward the oxidation of $o$-PD $(1 \mathrm{~mm} o$-PD, $1 \mathrm{~mm} \mathrm{H}_{2} \mathrm{O}_{2}, 5 \times 10^{-5} \mathrm{UmL}^{-1} \mathrm{HRP}$ ) in PBS of $\mathrm{pH}$ 6.0. Other membrane components (i.e., poly(vinyl chloride) (PVC) and $o$-NPOE in a weight ratio of 1:1) are the same for the six membrane electrodes.

shows the potential responses of $o$-PD oxidation on SPMs doped with different tetraphenylborate- and sulfonate-based receptors. Obviously, membranes doped with sodium salts of tetraphenylborate $\left(\mathrm{TPB}^{-}\right)$, tetra $\left(p\right.$-tolyl)borate $\left(\mathrm{TTB}^{-}\right)$, and tetrakis(4-fluorophenyl)borate $\left(\mathrm{TFPB}^{-}\right)$are superior than the dinonylnaphthalene sulfonate $\left(\mathrm{DNNS}^{-}\right)$-based membrane, which suggests better selectivities of these substituted tetraphenylborate-doped membranes toward $o$-PD oxidation intermediates. For SPMs sensitive to chemically stable alkylammonium monocations, substituted tetraphenylborates as ion receptors also yield superior sensitivity compared with the sulfonate additives. ${ }^{[38]}$ These better selectivities are probably related to the large aromatic $\pi$-systems in the tetraphenylborate structures, ${ }^{[39]}$ which lead to stronger noncovalent interactions with aromatic cations. However, the experimental measurement of these interactions was not attempted here owing to the difficulties in obtaining pure and stable intermediates. Unlike $\mathrm{TTB}^{-}$, $\mathrm{TPB}^{-}$and $\mathrm{TFPB}^{-}$, tetrakis[3,5bis(trifluoromethyl)phenyl]borate $\left(\mathrm{TTFPB}^{-}\right.$) and tetrakis[3,5-bis(1,1,1,3,3,3-hexafluoro-2-methoxy-2-propyl)phenyl]borate $\left(\mathrm{TFMPPB}^{-}\right)$used as the intermediate receptors yield potential responses even smaller than DNNS. This is likely because the bulky ortho-substitutents on phenyl groups may block their steric approach to organic intermediates.

Polymeric membranes plasticized with different kinds of water-immiscible solvents were compared. The polar solvent $o$-nitrophenyl octylether (o-NPOE) yields a membrane showing a more sensitive response toward $o$-PD oxidation as compared with dibutyl phthalate (DBP), bis(2-ethylhexyl) sebacate (DOS), and tris(2-ethylhexyl) phosphate (TEHP; Figure S3 in the Supporting Information), presumably due to the better solvation ability of $o$-NPOE for intermediate cations. Finally, the $o$-NPOE-PVC membrane doped with $\mathrm{TTB}^{-}$was employed in subsequent experiments, because it shows a sensitive potential response to $o$-PD oxidation but a small potential drift induced by $o$-PD (Figure 3 ). 

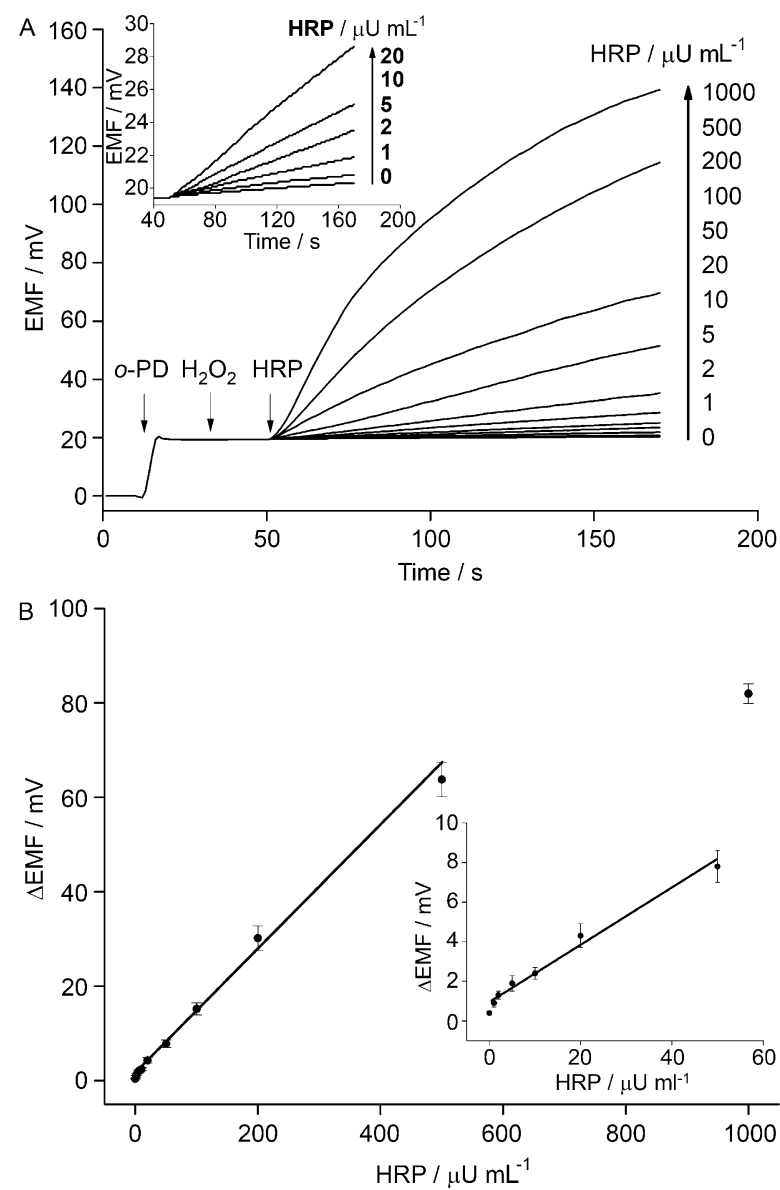

Figure 4. A) Potential traces for the $o$-PD oxidation ( $1 \mathrm{~mm} o$-PD, $1 \mathrm{~mm}$ $\mathrm{H}_{2} \mathrm{O}_{2}$ ) catalyzed by different activities of HRP in PBS (50 mM) of $\mathrm{pH}$ 6.0. B) Calibration curve for HRP. Each error bar represents one standard deviation for three measurements.

Figure $4 \mathrm{~A}$ shows the potential responses of the $o$-PD oxidations catalyzed by various activities of HRP in PBS $(50 \mathrm{~mm})$ of $\mathrm{pH}$ 6.0. The potential increases measured at one minute after the initiation of $o$-PD oxidation were used for quantification. As shown in Figure 4B, HRP can be detected with a linear range of $1 \times 10^{-6}$ to $5 \times 10^{-4} \mathrm{U} \mathrm{mL}^{-1}$ and a detection limit of $4 \times 10^{-7} \mathrm{UmL}^{-1}(3 \sigma)$. The detection limit is three orders of magnitude lower than those obtained by HRP sensing protocols based on classical ion-selective membrane electrodes ${ }^{[17 a-c]}$ and two orders of magnitude lower than that by the spectrophotometric method under identical reaction conditions (Figure S4 in the Supporting Information). Notably, this is a preliminary investigation demonstrating the feasibility of the intermediate-sensitive SPM in potentiometric determination of peroxidase activities and its basic characteristics. In fact, there is a very large space for improvement of the sensitivity. For example, the use of fluorous membrane allowing for especially strong ion pair formation and weak interfering ion solvation could elevate the selectivities toward alkylammonium cations over inorganic ions by several orders of magnitude ${ }^{[40]}$ which may also yield intriguing sensitivities for organic intermediates in the $o$-PD oxidation. Since potential responses of intermediates are dictated by the ion exchange process across the membranesample interface, the sensitivity could be improved by use of more rigid membrane matrix such as poly(acrylates) to suppress the target ion diffusion into the membrane bulk ${ }^{[41]}$ or a rotating electrode configuration to enhance the transport of target ion from the sample solution into the sample-membrane interface. ${ }^{[42]}$

The regenerability of the intermediate-sensitive SPME is important for its practical use. For $o$-PD oxidation catalyzed by HRP even in the upper end of the dynamic range, a 2 min washing step readily regenerates the electrode (Figure S5 in the Supporting Information). With ten consecutive measurements using the same electrode, the standard derivation of potential responses in the first minute is $2.9 \%$. This reversibility is much better than the previously reported $N_{4}$-TMB oxidation system, in which the regeneration of an electrode requires at least 20 minutes even with very low reactant concentrations. ${ }^{[18]}$ Such a discrepancy is reasonable in view of the extraordinary strong ion association interaction of DNNS with the doubly charged diimine intermediate in $N_{4}$-TMB oxidation (Figure S6 in the Supporting Information) ${ }^{[18]}$ In contrast, $o$-PD intermediates are mainly monocationic under the employed conditions according to their $\mathrm{p} K_{\mathrm{a}}$ values for the second protonation (data not shown), thereby possessing much smaller ion association constants with counterions as compared with dications. ${ }^{[37,43]}$

In addition to the high sensitivity and excellent reversibility, the present peroxidase detection method has other advantages. The SPM functionalized with a substituted tetraphenylborate show lower membrane resistance as compared with the previously used DNNS-doped membrane, ${ }^{[18,44]}$ which yields smaller potential noises and facilitates its miniaturization. In addition, in contrast to $N_{4}$-TMB, whose stock solution is difficult to prepare (long-time sonication is needed even when using DMSO as solvent), the preparation of the $o$-PD stock solution is easy by using ethanol as solvent.

Application of the intermediate-sensitive membrane in Gquadruplex/hemin DNAzyme-based biosensing: To examine the feasibility of the intermediate-sensitive SPM in probing peroxidase mimetics, the G-quadruplex/hemin DNAzyme was employed for its wide applications in sensing of DNA, proteins, enzymes, small molecules, and metal ions. ${ }^{[2 \mathrm{~b}, 45]}$ As shown in Figure 5, a large potential response can be obtained with a model G-quadruplex/hemin DNAzyme as the catalyst of the $\mathrm{H}_{2} \mathrm{O}_{2}$-mediated $o$-PD oxidation, whereas a negligible response is induced when G-quadruplex or hemin is present alone. Notably, a buffer of $\mathrm{pH} 7.5$ was used because the intermediate-sensitive SPME was found to show the most sensitive response at this $\mathrm{pH}$ for the DNAzymecatalyzed $o$-PD oxidation, which is different from the mildly acidic optimal pH for HRP (see Figure S7 in the Supporting Information).

As a preliminary application, a potentiometric label-free thrombin detection protocol based on a thrombin-binding 


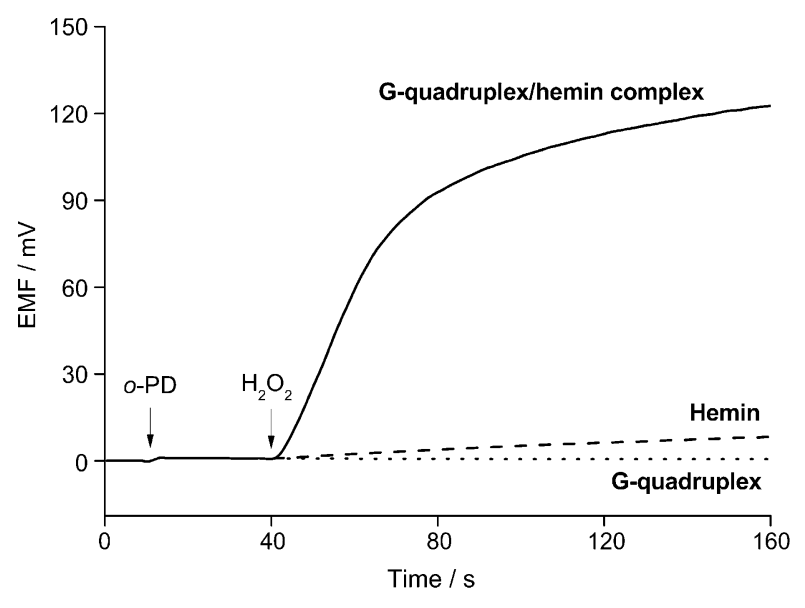

Figure 5. Potential responses of the $\mathrm{H}_{2} \mathrm{O}_{2}$-mediated oxidation of $o$-PD catalyzed by the G-quadruplex (5'-GGGTAGGGCGGGTTGGGT-3')/ hemin complex ${ }^{[46]}$ hemin, and G-quadruplex. Experimental conditions: HEPES buffer $(20 \mathrm{~mm})$, pH 7.5, G-rich DNA $(0.5 \mu \mathrm{M})$, hemin $(0.5 \mu \mathrm{M}), o$ $\mathrm{PD}(1 \mathrm{~mm}), \mathrm{H}_{2} \mathrm{O}_{2}(1 \mathrm{~mm})$. Before measurements, the catalysts were incubated in the HEPES buffer solution for $1 \mathrm{~h}$.

aptamer was developed. Although the thrombin-binding aptamer itself could fold into a G-quadruplex and display peroxidatic activity in the presence of hemin and $\mathrm{K}^{+}$, this activity would be enhanced upon the binding of thrombin-binding aptamer with the target thrombin. ${ }^{[47]}$ As shown in Figure 6, in the presence of thrombin, larger potential responses can be obtained with the intermediate-sensitive SPME as compared with that of the thrombin-binding aptamer/hemin complex alone. In the range of 2 to $50 \mathrm{~nm}$, the thrombin concentration shows a linear relationship with the potential change measured at $5 \mathrm{~min}$ after initiation of the catalyzed oxidation. Moreover, interfering proteins including bovine serum albumin, lysozyme, and IgG do not en-

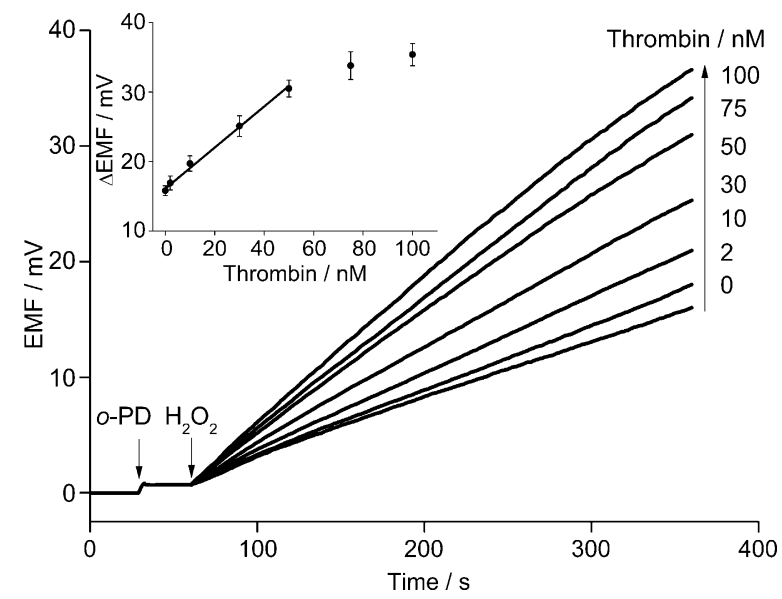

Figure 6. Potential traces for $o$-PD oxidation catalyzed by the supramolecular complexes of the thrombin-binding aptamer, hemin, and thrombin at different concentrations. Experimental conditions: HEPES buffer (20 mM), pH 7.5, thrombin-binding aptamer, $(2 \mu \mathrm{M})$, hemin $(2 \mu \mathrm{M}), o$-PD (1 mM), $\mathrm{H}_{2} \mathrm{O}_{2}(1 \mathrm{~mm})$. The inset shows the calibration curve for thrombin detection. hance the peroxidatic activity of thrombin-binding aptamer even at a high level of $100 \mathrm{~nm}$ (data not shown), which indicates the specificity of this sensing protocol toward thrombin. In addition to thrombin, the proposed potentiometric technique is expected to detect other analytes (e.g., targets of G-quadruplex-aptamer conjugates) by using corresponding G-quadruplex DNAzyme-involved biorecognition systems.

\section{Conclusion}

Reaction intermediates with different $\mathrm{p} K_{\mathrm{a}}$ values from those of the substrates and final products have been utilized to develop potentiometric biosensing protocols. By using the potential response of $o$-PD oxidation intermediates on the substituted tetraphenylborate-doped SPME, horseradish peroxidase and peroxidase-mimicking DNAzymes can be sensitively detected. With advantages such as high sensitivity, excellent regenerability, simple instrumentation, and low cost, this intermediate-sensitive membrane electrode promises to be a competitive transducer for use in biosensing schemes based on peroxidase and its mimetics, and to serve as a facile technique for screening nanomaterials with peroxidase activities. In addition to efforts on further applications of the proposed membrane electrode (e.g., in peroxidase nanomimetic-based biosensing), we are exploring more analytically important enzymatic reactions suitable for membrane detection by using the intermediate-based sensing methodology.

\section{Experimental Section}

Reagents and materials: BHA, sodium tetraphenylborate, sodium tetrakis(4-fluorophenyl)borate dihydrate, sodium tetra( $p$-tolyl)borate, sodium tetrakis[3,5-bis(trifluoromethyl)phenyl]borate, sodium tetrakis[3,5bis(1,1,1,3,3,3-hexafluoro-2-methoxy-2-propyl)phenyl]borate trihydrate, dinonylnaphthalene sulfonic acid as a $50 \%$ solution in heptane, $o$-NPOE, DBP, DOS, TEHP, high molecular weight poly(vinyl chloride), peroxidase from horseradish (Type VI, essentially salt-free, lyophilized powder, $256 \mathrm{U} \mathrm{mg}^{-1}$ solid), and thrombin from human plasma (2164 $\mathrm{U} \mathrm{mg}^{-1}$ ) were purchased from Sigma-Aldrich. The 15-mer thrombin-binding aptamer (5'-GGTTGGTGTGGTTGG-3') and a model G-quadruplex (5'GGGTAGGGCGGGTTGGGT-3') were synthesized by Shanghai Sunny Biotech Co., Ltd. Other reagents were purchased from the Sinopharm Group Co., Ltd. All chemicals were of Selectophore or analytical reagent grade. Aqueous solutions were prepared with freshly deionized water (18.2 $\mathrm{M} \Omega \mathrm{cm}$ specific resistance) obtained with a Pall Cascada laboratory water system.

Membrane electrode preparation and EMF measurements: Polymeric membranes containing PVC and plasticizer in a weight ratio of 1:1 and a cation exchanger $\left(25 \mathrm{~mm} \mathrm{~kg}^{-1}\right)$ were prepared by using a solvent-casting technique with tetrahydrofuran as the casting solvent. Disks of $5 \mathrm{~mm}$ diameter were punched from the parent membrane and glued to plasticized PVC tubes (i.d. $3 \mathrm{~mm}$, o.d. $5 \mathrm{~mm}$ ) to fabricate the polymeric membrane electrodes. For peroxidase measurements, the electrodes were conditioned, overnight, in $\mathrm{NaCl}(15 \mathrm{~mm})$ and the same solution was used as the inner filling medium. For DNAzyme measurements, HEPES buffer of pH $7.5(20 \mathrm{~mm} N$-2-hydroxyethylpiperazine- $N$-ethane sulfonic acid 
(HEPES), $150 \mathrm{~mm} \mathrm{NaCl}, 20 \mathrm{~mm} \mathrm{KCl,} 50 \mathrm{~mm} \mathrm{NH} 4 \mathrm{OAc}$ ) was used as the conditioning and inner filling solution.

All electromotive force (EMF) values were measured by using a CHI $760 \mathrm{D}$ electrochemical workstation (Shanghai Chenhua Apparatus Corporation, China) at room temperature in the following galvanic cell in a Faraday cage: $\mathrm{Ag}, \mathrm{AgCl} / \mathrm{KCl}(3 \mathrm{M}) / \mathrm{LiOAc}(1 \mathrm{~m}) /$ reaction solution (wellstirred)/sensing membrane/inner filling solution/ $\mathrm{AgCl}, \mathrm{Ag}$. The external reference electrode was made as follows: a $100 \mu \mathrm{L}$ pipette tip with its narrower end obstructed by plastic foam was first filled with $3 \mathrm{M} \mathrm{KCl}$; then an $\mathrm{Ag} / \mathrm{AgCl}$ wire was inserted into this pipette tip and the other end of the pipette tip was sealed with Parafilm.

Electrospray ionization mass spectrometry (ESI/MS) measurements: ESI/MS analyses were performed on a LCQ Fleet ion trap mass spectrometer (Thermo Finnigan) in positive ion mode. Samples were loop-injected into the ESI source with a syringe and a carrier flow of methanol at a flow rate of $200 \mu \mathrm{L} \mathrm{min}^{-1}$ was employed. The instrumental conditions were as follows: sheath gas flow rate, 20 units; aux gas flow rate, 5 units; spray voltage, $5 \mathrm{kV}$; capillary temperature, $300^{\circ} \mathrm{C}$; capillary voltage, $35 \mathrm{~V}$; tube lens, $110 \mathrm{~V}$. All mass spectra were blank-subtracted.

Thrombin detection with the intermediate-sensitive membrane: The procedures for the formation of thrombin/thrombin-binding aptamer/hemin supramolecular complex were similar to those reported elsewhere. ${ }^{[47]}$ Briefly, thrombin-binding aptamer $(30 \mu \mathrm{L}, 20 \mu \mathrm{M})$ in TE buffer $(10 \mathrm{~mm}$ Tris- $\mathrm{HCl}, \mathrm{pH} 7.4,0.1 \mathrm{~mm}$ EDTA) was heated at $88^{\circ} \mathrm{C}$ for $10 \mathrm{~min}$ and gradually cooled to room temperature. Then, $30 \mu \mathrm{L}$ of the thrombinbinding buffer solution (Tris- $\mathrm{HCl}(20 \mathrm{~mm}), \mathrm{NaCl}(140 \mathrm{~mm}), \mathrm{KCl}(5 \mathrm{~mm})$, $\mathrm{CaCl}_{2}(1 \mathrm{~mm}), \mathrm{MgCl}_{2}$ ( $\left.1 \mathrm{~mm}\right), \mathrm{pH}$ 7.4) containing thrombin was added to the thrombin-binding aptamer solution. After $3 \mathrm{~h}$ incubation at room temperature, hemin $(30 \mu \mathrm{L}, 20 \mu \mathrm{M})$ in HEPES buffer was added to the mixture to interact for another $2 \mathrm{~h}$. Finally, the resulting thrombin/aptamer/hemin complex solution was diluted to $300 \mu \mathrm{L}$ with HEPES buffer and was tested by the intermediate-sensitive SPME.

\section{Acknowledgements}

We thank Prof. Ilario Losito, University of Bari "Aldo Moro", for helpful discussions on sequential MS and Dr. Ying Liu for her technical support for ESI-MS measurements. This work was financially supported by the Instrument Developing Project of the Chinese Academy of Sciences (YZ201161), the National Natural Science Foundation of China (41176081), and the Taishan Scholar Program of Shandong Province (TS20081159)

[1] J. X. Xie, X. D. Zhang, H. Wang, H. Z. Zheng, Y. M. Huang, $\operatorname{Tr} A C$ Trends Anal. Chem. 2012, 39, 114-129.

[2] a) T. T. Ngo, Anal. Lett. 2010, 43, 1572-1587; b) L. Lv, Z. J. Guo, J. H. Wang, E. K. Wang, Curr. Pharm. Des. 2012, 18, 2076-2095; c) J. Li, F. Jiang, X. Wei, Anal. Chem. 2010, 82, 6074-6078.

[3] Y. Song, K. Qu, C. Zhao, J. Ren, X. Qu, Adv. Mater. 2010, 22, 22062210.

[4] N. Ding, N. Yan, C. Ren, X. Chen, Anal. Chem. 2010, 82, 58975899.

[5] M. Liang, K. Fan, Y. Pan, H. Jiang, F. Wang, D. Yang, D. Lu, J. Feng, J. Zhao, L. Yang, X. Yan, Anal. Chem. 2013, 85, 308-312.

[6] a) Y. Song, X. Wang, C. Zhao, K. Qu, J. Ren, X. Qu, Chem. Eur. J. 2010, 16, 3617-3621; b) Y. J. Guo, L. Deng, J. Li, S. J. Guo, E. K. Wang, S. J. Dong, ACS Nano 2011, 5, 1282-1290; c) Y. Song, Y. Chen, L. Feng, J. Ren, X. Qu, Chem. Commun. 2011, 47, 44364438.

[7] a) S. Liu, F. Lu, R. Xing, J. J. Zhu, Chem. Eur. J. 2011, 17, 620-625; b) X. Yang, C. Fang, H. Mei, T. Chang, Z. Cao, D. Shangguan, Chem. Eur. J. 2011, 17, 14475-14484.

[8] a) W. Luo, Y. S. Li, J. Yuan, L. H. Zhu, Z. D. Liu, H. Q. Tang, S. S Liu, Talanta 2010, 81, 901-907; b) B. Qiu, Z. Z. Zheng, Y. J. Lu,
Z. Y. Lin, K. Y. Wong, G. N. Chen, Chem. Commun. 2011, 47, $1437-$ 1439.

[9] W. B. Shi, X. D. Zhang, S. H. He, Y. M. Huang, Chem. Commun. 2011, 47, 10785-10787.

[10] X. Zheng, Q. Liu, C. Jing, Y. Li, D. Li, W. Luo, Y. Wen, Y. He, Q. Huang, Y. T. Long, C. Fan, Angew. Chem. 2011, 123, 12200-12204 Angew. Chem. Int. Ed. 2011, 50, 11994-11998.

[11] E. Bakker, E. Pretsch, Angew. Chem. 2007, 119, 5758-5767; Angew. Chem. Int. Ed. 2007, 46, 5660-5668.

[12] P. Buhlmann, L. D. Chen in Supramolecular Chemistry: From Molecules to Nanomaterials, Vol. 5 (Eds.: P. A. Gale, J. W. Steed), Wiley, New York, 2012, pp. 2539-2579.

[13] a) O. T. Guenat, S. Generelli, N. F. de Rooij, M. Koudelka-Hep, F. Berthiaume, M. L. Yarmush, Anal. Chem. 2006, 78, 7453-7460; b) M. H. Asif, A. Fulati, O. Nur, M. Willander, C. Brannmark, P. Stralfors, S. I. Borjesson, F. Elinder, Appl. Phys. Lett. 2009, 95, 023703.

[14] a) J. W. Ding, W. Qin, Chem. Commun. 2009, 971-973; b) M. Cuartero, J. A. Ortuño, M. S. García, F. García-Cánovas, Anal. Biochem. 2012, 421, 208-212.

[15] J. Szúcs, E. Pretsch, R. E. Gyurcsányi, Analyst 2009, 134, 1601-1607.

[16] a) H. S. Abd-Rabboh, S. A. Nevins, N. Dürüst, M. E. Meyerhoff, Biosens. Bioelectron. 2003, 18, 229-236; b) X. W. Wang, Q. Wang, W. Qin, Biosens. Bioelectron. 2012, 38, 145-150.

[17] a) J. L. Boitieux, G. Desmet, D. Thomas, Clin. Chem. 1979, 25, 318 321; b) P. W. Alexander, C. Maltra, Anal. Chem. 1982, 54, 68-71; c) D. C. Cowell, A. A. Dowman, J. D. Newman, R. Pirzad, C. Rantle, Biosens. Bioelectron. 1992, 7, 27-38; d) X. W. Wang, Z. F. Ding, Q. W. Ren, W. Qin, Anal. Chem. 2013, 85, 1945-1950.

[18] X. W. Wang, W. Qin, Chem. Commun. 2012, 48, 4073-4075.

[19] a) P. J. Tarcha, V. P. Chu, D. Whittern, Anal. Biochem. 1987, 165, 230-233; b) K. Jiao, G. Sun, S. S. Zhang, Sci. China Ser. B 1998, 41, 245-352.

[20] S. Fornera, P. Walde, Anal. Biochem. 2010, 407, 293-295.

[21] V. M. Mekler, S. M. Bystryak, Anal. Chim. Acta 1992, 264, 359-363.

[22] L. Y. Hou, Z. J. Zhang, L. R. Luo, Anal. Bioanal. Chem. 2007, 387, 925-931.

[23] a) L. Fruk, J. Müller, G. Weber, A. Narváez, E. Domínguez, C. M. Niemeyer, Chem. Eur. J. 2007, 13, 5223-5231; b) K. Liu, J. J. Zhang, C. Wang, J. J. Zhu, Biosens. Bioelectron. 2011, 26, 3627-3632.

[24] K. C. Brown, J. F. Corbett, N. P. Loveless, Spectrochim. Acta Part A 1979, 35, 421-423.

[25] D. Schomberg, M. Salzmann, D. Stephan in Enzyme Handbook 7, EC 1. 11. 1. 7:1-6, Springer, Berlin, 1993

[26] P. Vetešník, J. Bielavský, M. Kaválek, M. Veeřa, Collect. Czech. Chem. Commun. 1968, 33, 2902-2910.

[27] H. B. Dunford in Peroxidases in Chemistry and Biology, Vol. 2 (Eds.: J. Everse, K. E. Everse, M. B. Grisham), CRC, Boca Raton, 1991, pp. $1-24$.

[28] I. Losito, N. Cioffi, M. P. Vitale, F. Palmisano, Rapid Commun. Mass Spectrom. 2003, 17, 1169-1179.

[29] R. M. Uppu, G. L. Squadrito, R. M. Bolzan, W. A. Pryor, J. Am. Chem. Soc. 2000, 122, 6911-6916.

[30] a) A. Claiborne, I. Fridovich, Biochemistry 1979, 18, 2324-2329; b) P. D. Josephy, R. P. Mason, T. Eling, Carcinogenesis 1982, 3 , 1227-1230; c) P. D. Josephy, A. Van Damme, Biochem. Pharmacol. 1984, 33, 1155-1156.

[31] J. Kaizer, R. Csonka, G. Speier, React. Kinet. Catal. Lett. 2002, 75, $367-374$.

[32] C. Hempen, S. M. Van Leeuwen, H. Luftmann, U. Karst, Anal. Bioanal. Chem. 2005, 382, 234-238.

[33] a) T. Nogami, T. Hishida, M. Yamada, H. Mikawa, Y. Shirota, Bull. Chem. Soc. Jpn. 1975, 48, 3709-3714; b) J. F. Corbett, S. Pohl, I. Rodriguez, J. Chem. Soc. Perkin Trans. 2 1975, 728-734.

[34] For $\mathrm{p} K_{\mathrm{a}}$ values of $o-\mathrm{PD}$ and DAP, see refs. [24] and [26]. For $\mathrm{p} K_{\mathrm{a}}$ of $o$-PD radical, see: M. Jonsson, J. Lind, G. Merényi, T. E. Eriksen, $J$. Chem. Soc. Perkin Trans. 2 1995, 2, 61-65. Other $\mathrm{p} K_{\mathrm{a}}$ values were calculated with ChemAxon's Marvin: http://www. chemaxon.com/ demosite/marvin/index.html; accessed August 7, 2012; its accuracy 
in predicting $\mathrm{p} K_{\mathrm{a}}$ values of benzoquinone diimine structures was confirmed in the Supporting Information.

[35] J. F. Corbett, J. Chem. Soc. B 1969, 827-835.

[36] J. F. Corbett, E. P. Gamson, J. Chem. Soc. Perkin Trans. 2 1972, $1531-1537$.

[37] T. Alaviuhkola, J. Bobacka, M. Nissinen, K. Rissanen, A. Ivaska, J. Pursiainen, Chem. Eur. J. 2005, 11, 2071-2080.

[38] V. Egorov, A. Bolotin, J. Anal. Chem. 2006, 61, 279-283.

[39] P. K. Bakshi, A. Linden, B. R. Vincent, S. P. Roe, D. Adhikesavalu, T. S. Cameron, O. Knop, Can. J. Chem. 1994, 72, 1273-1293.

[40] P. G. Boswell, P. Bühlmann, J. Am. Chem. Soc. 2005, 127, $8958-$ 8959.

[41] a) L. Y. Heng, K. Toth, E. A. H. Hall, Talanta 2004, 63, 73-87; b) F. Sundfors, T. Lindfors, L. Höfler, R. Bereczki, R. E. Gyurcsányi, Anal. Chem. 2009, 81, 5925-5934.

[42] a) Q. S. Ye, M. E. Meyerhoff, Anal. Chem. 2001, 73, 332-336; b) W. Qin, W. Zhang, K. P. Xiao, M. E. Meyerhoff, Anal. Bioanal. Chem.
2003, 377, 929-936; c) W. Qin, R. N. Liang, X. L. Fu, Q. W. Wang, T. J. Yin, W. J. Song, Anal. Chem. 2012, 84, 10509-10513.

[43] H. R. Sun, J. C. Biffinger, S. G. DiMagno, Dalton Trans. 2005, 3148 3154.

[44] N. Ramamurthy, PhD Thesis, University of Michigan, Ann Arbor, 1999.

[45] I. Willner, B. Shlyahovsky, M. Zayats, B. Willner, Chem. Soc. Rev. 2008, 37, 1153-1165.

[46] S. Nakayama, J. Wang, H. O. Sintim, Chem. Eur. J. 2011, 17, 56915698.

[47] a) T. Li, E. Wang, S. Dong, Chem. Commun. 2008, 3654-3656; b) Y. Zhang, B. Li, Y. Jin, Analyst 2011, 136, 3268-3273.

Received: January 25, 2013 Revised: April 28, 2013 Published online: June 12, 2013 